\title{
An Extended Locking Method for Geographical Database with Spatial Rules
}

\author{
Changxiu Cheng ${ }^{1}$, Paiwei Shen ${ }^{1}$, Mingbo Zhang ${ }^{1,2}$, and Feng Lu ${ }^{1}$ \\ ${ }^{1}$ State Key Laboratory of Resources and Environmental Information System, \\ Institute of Geographical Sciences and Natural Resources Research, \\ Chinese Academy of Sciences, Beijing 100101, P.R.China \\ ${ }^{2}$ Shan Dong University of Technology, Zi Bo 255000,P.R China
}

\begin{abstract}
Locking and unlocking operations are crucial to keep data consistency for concurrency control. Compared with traditional pessimistic locking method, the neutral concurrency control scheme can highly improve the concurrency of spatial database management systems. However, in consideration of spatial rules, dead locks brought about by waiting mutually for releasing required resources may frequently arise. To avoid such dead locks, referring to the theories of session management in operation system, we set forward an extended locking method for geographical database with spatial rules to implement the collaboration and competition between concurrent locking and unlocking transactions. The method avoids the expenditure of detecting dead locks and rolling back, and the system becomes more efficient. The test shows the extended locking technique makes the spatial database management system more ordered and efficient in a multi-users environment only with some time consumption of managing logical codes.
\end{abstract}

\section{Introduction}

HYPERLINKA heterogeneous, distributed, and loose-coupled working environment is evolving. The cooperative GIS will be popular in the future. As a key technology to realize cooperative GIS, concurrency control is also emerging, i.e. how to ensure that geographical database operations from different users do not interfere with each other (Hanssen 2003).

The early GIS were based on CAD systems where the map base was held as a collection of CAD drawings. The methods of managing such a system where multiple users wish to access and update the map base are based on the manual drawing office approach. Indeed there is a market for Document Management systems(Newell 2003).However, as GIS shift to Relation Database Management Systems form Document Management systems, the concurrency control scheme of GIS has been changed. Most distributed database employ either pessimistic or optimistic concurrency control scheme ( Lee and Yang et al 2000 ). The former often results in the high communication delay, because the spatial data queried by clients would be hold for a long time. Generally, the latter is employed in distributed GIS application, i.e. version management. The version management in GIS has been put forward for 
decades (Easterfield and Newell et al 1990). Now, it has been widely used, i.e. ArcSDE (Gaskill and Brooks 2000), Oracle Spatial (Arun 2001). But it makes the system complex and perplexes users with undoing and redoing of previous actions on user interface when conflict occurs ( Lee and Yang et al 2000, Batty 2002 ). In addition, the version management is not reasonable to distribute interactive GIS applications. In this application, the changes are not so complex, the changed entities are not so many, but the results are required to submit in a few minutes. WAVE (Lea and Matsuda 1997) adopts a simple locking mechanism for this interactive application. A user locks objects that would be manipulated and updates them. The updates are propagated to the other users while the lock is released. It was named Neutral Concurrency Control Scheme (NCCS) in this paper.

However, only locking these manipulated spatial entities is not enough considering spatial rule-based processing (Michael 2001, Mayal and Hall 2002, Sha and Bian 2002)in NCCS. So, not only these manipulated objects but also their neighboring objects should be locked in geographical database with spatial rules. Then it will be frequent to encounter dead locks resulted by waiting for releasing required resources by each other. To avoid the emergence of such dead locks, we propose an extended locking method for spatial rule-based processing. It makes the spatial database management system more ordered and efficient in a multi-users environment only with some time consumption of managing logical codes.

The remaining parts of this paper are organized as follows. Section 2 describes the neutral concurrency control scheme and introduces why the dead lock are frequently encountered in spatial rule-based geographical database. Section 3 analyzes the reason why dead locks occur, and presents an extended locking method to avoid the emergence of such dead locks. The implementation of the extended locking method in Oracle Spatial is then introduced in section 4. Section 5 bring forward two tests, which show that the extended locking method makes the spatial database management system more ordered and efficient in a multi-users environment with just some time consumption of managing logical codes. Finally, in Section 6 of this paper, summary as well as forward are made as conclusion.

\section{Neutral Concurrency Control Scheme and Deadlocks}

In pessimistic concurrency control scheme, there are two types of locks. One is share lock (lock-S); another is exclusive lock (lock-X). Share lock specifies that others may read while you read, but writing objects in the storage group is prevented. Several users can have the share lock at the same time. Exclusive lock prevents other users from getting either of the two kinds of locks. In NCCS, there are also share lock and exclusive lock. But there is something different in compatibility matrix. The share lock allows that one and only one user should write objects while you read. In order to keep consistency, the writing results are propagated to the other users when the exclusive lock is released.

However, only locking these manipulated spatial entities is not enough considering spatial rule-based processing in NCCS. If fig 1 conforms to share edit rule, the shape of entity $C$, entity D and entity $G$ will be changed on moving the $\mathrm{P}_{1} \mathrm{P}_{2}$ of entity $\mathrm{A}$. 
Therefore, in spatial rule-based geographical database, not only these manipulated objects but also their neighboring objects should be locked. Because the locking granularity is not atomic, a deadlock will occur when two or more users are waiting for data unlocked by each other. If transaction 1 and transaction 2 are executed according to the schedule shown in fig 2, transaction 1 will be choked and wait for transaction 2 to unlock the entity D, and transaction 2 will also be choked and wait for transaction 1 to unlock the entity $\mathrm{C}$ at the time point of T5. As a result, the deadlock occurs.

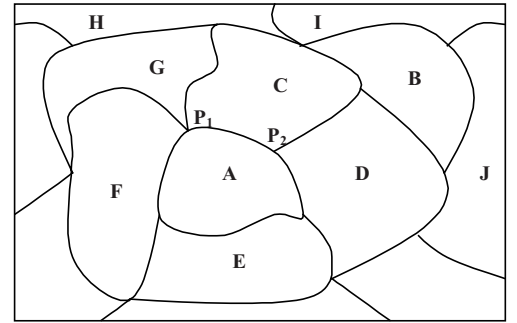

Fig. 1. Map view

\begin{tabular}{|c|c|c|}
\hline & $\begin{array}{c}\text { Transaction 1 : } \\
\text { Edit A Entity }\end{array}$ & $\begin{array}{c}\text { Transaction 2 : } \\
\text { Edit B Entity }\end{array}$ \\
\hline T0 & Begin & \\
\hline T1 & Lock-X C & \\
\hline T2 & Lock-X G & Begin \\
\hline T3 & Lock-X F & Lock-X J \\
\hline T4 & Lock-X E & Lock-X D \\
\hline T5 & Lock-X D & Lock-X C \\
\hline$\ldots$ & $\ldots$ & $\ldots$ \\
\hline T (n-1) & Edit & Edit \\
\hline Tn & Commit & Commit \\
\hline
\end{tabular}

Fig. 2. The executive schedule

In geographical database with spatial rule-based, deadlocks occur most often. Because there are many kinds of spatial rule-based in GIS, and some rules maybe lock many entities. For example, when a user is editing the "Changjiang River" of china, he will hold the many spatial data that cover large areas, and then the probability of the occurrence of deadlocks would be high. Some time the deadlocks are no less than the chain reaction specified in the multi-user environment.

\section{The Extended Locking Method}

To avoid the emergence of such dead locks, referring to the theories of session management in operation system, we set forward an extended locking method to implement the collaboration and competition relationships between locking and unlocking transactions.

\subsection{The Collaboration between Locking and Unlocking Transactions}

Collaboration is the style or approach used by some processes as they work toward a common goal. Locking, updating, and unlocking are three basic steps in any updating transaction. In the updating transaction, the systems must make them work according to some rules. They are as follows: Rule 1: Firstly lock the data that would be updated. Then update them. At last unlock them. Rule 2: the lock operation must be atomic. Ensures that either all lock operations of a transaction accomplish successfully or all of its effects fail. Rule 3: the lock operations have to wait until others unlock the Data. So, it is a type of collaboration. 
The data synchronization is an effective instrument to implement the collaboration. We can set an IsXLocked signal to any spatial entity, which is used for communication. The entity is not locked by transaction, if its IsXLocked equals 0 . And it is locked by transaction, if its IsXLocked equals 1. According to the rules of updating transaction, the locking and unlocking logic can be presented as follows shown in fig 3. The UpdateTransaction in fig3 could avoid some deadlocks.

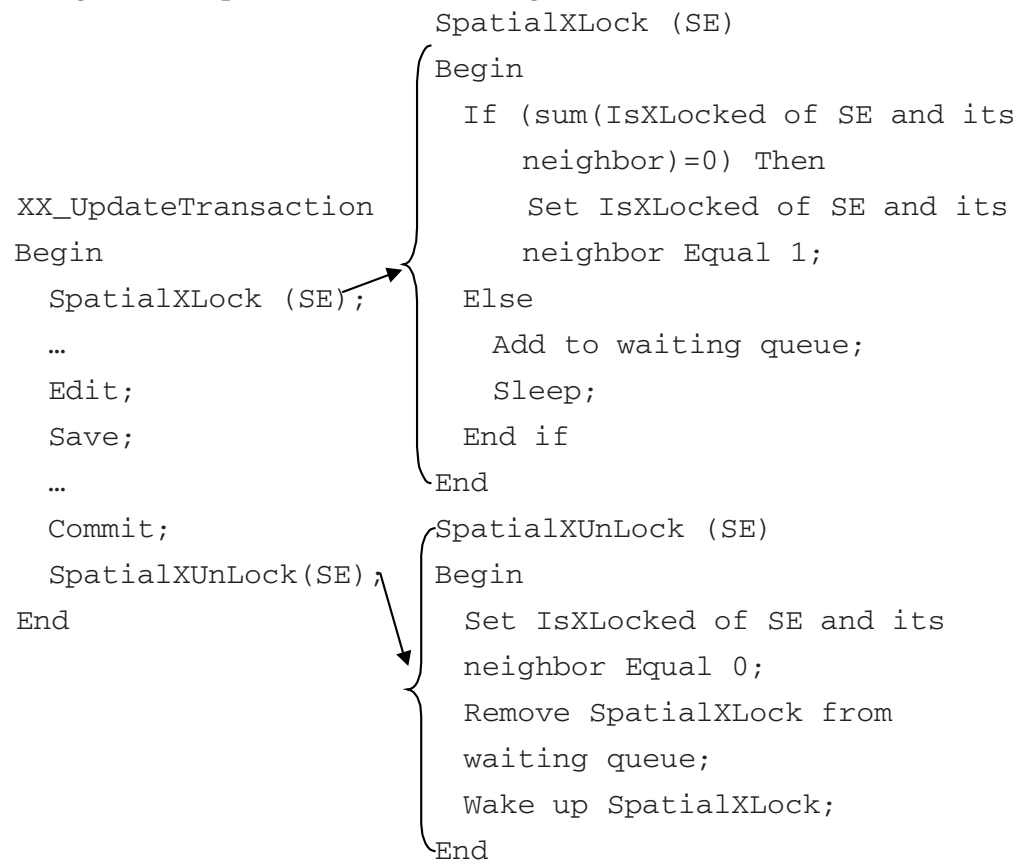

Fig. 3. Logic of locking and unlocking

\subsection{The Competition between Locking and Unlocking Transactions}

Accessing the shared resources causes the competition between locking and unlocking transactions. In the multi-user environment, many locking transactions exist at the same time. These transactions maybe request the same resource, change the same data, and keep the change for a period. Then the shared data may be inconsistent, and deadlocks will be resulted from. For example, as shown in fig 2, if transaction 1 executes the if clause in fig 3 at time point T0 and transaction 2 executes the if clause at time point T2, the IsXLocked value of the entity $\mathrm{C}$ will still be 0 at time point T2, because its value will not change until the transaction is committed. So, transaction 2 will not be chocked, and the deadlocks still occurred at time point T5.

In order to keep consistency of share variables, the locking and unlocking transactions must be mutex. We can define the code section that access IsXLocked as critical section (CS) that only allows one transaction to enter this code section at the same time. The semaphore, SemWait and SemSignal operations can implement the 
concept of CS. A semaphore called Mutex, is integer variables that, apart from initialization, can be accessed only through atomic SemWait and SemSignal operations. If insert the SemWait operations after Begin clause of locking and unlocking transaction, and insert the SemSignal operations after Begin clause of them, the locking and unlocking transactions will be mutually exclusive.

\section{The Implementation of Extended Locking Method}

There are two troublesome in the implementation of extended locking method in Oracle Spatial. One is the implementation of critical section; another is transaction queue mechanism. There isn't the concept of critical section in PL/SQL of Oracle, so it must be simulated with other techniques. And the advanced queue is very complex, so coding it is difficult.

In Oracle, the exclusive table lock is the most restrictive mode of table lock. Only one transaction can obtain an exclusive table lock for a table.So, if we create Table_mutex table as semaphore, then the SemWait operation (EnterCritical Section ) can be programmed as follows:

Begin

LOCK TABLE Table_Mutex IN exclusive MODE; --Exclusive Lock

End

However, it is needless to program the SemSignal operation, because it operation has been included in commit clause at the end of transaction. The commit will release the exclusively hold of Table_Mutex, and wake up some waiting transactions. So, it is obvious that the queue mechanisms has worked in the lock table and commit clause.

Given the geometry column was name 'shape', we can add IsXLocked column as signal to express the entities whether locked or unlocked. Then, the locking transaction can be programmed as followed:

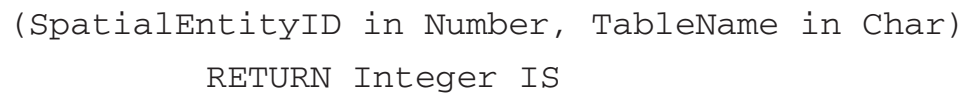

\section{BEGIN}

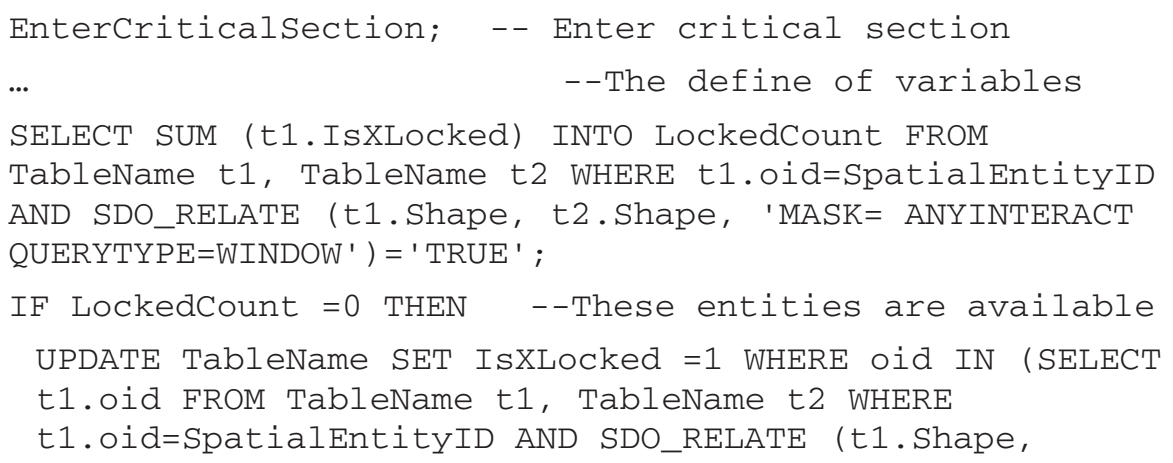




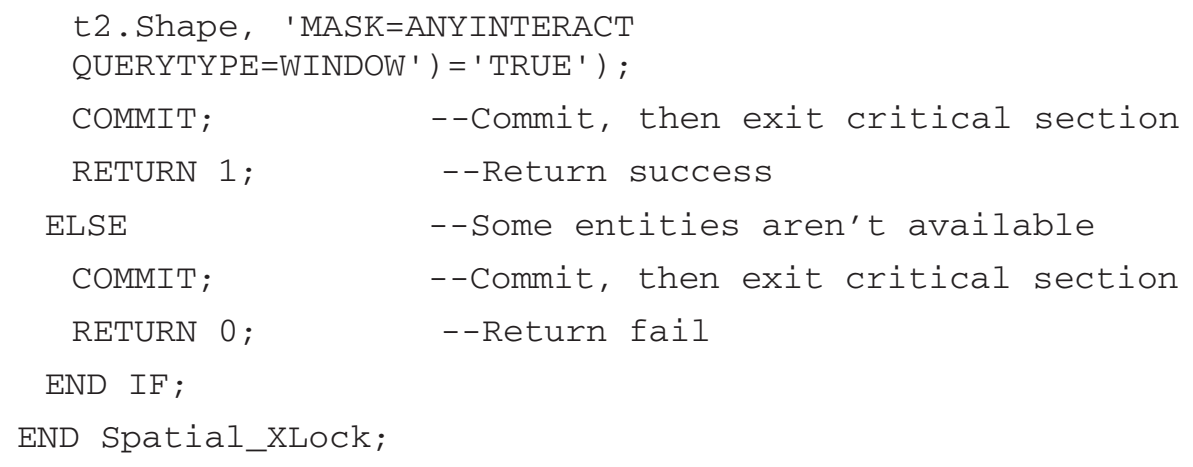

END IF ;

\section{END Spatial_XLock;}

The produce is not optimum, because the implicit spatial query of anyinteract is executed twice. We can define some arrays in which the results of the spatial query are stored, which will be reusable. Additionally, the explicit query also can make the produce more effective than implicit query. The unlocking transaction is simpler than the locking transaction; details of which will not be presented here in this paper.

\section{Tests and Performance Analysis}

The test data is the chinese county boundary scale at 1:4,000,000, in which there are 2525 polygon. The database management systems is Oracle $9 \mathrm{i}$. The database server is Dell4600.

Test1:Set up only one session (single user environment), use the old method described in section 3 to lock and unlock these polygons one by one, and record the elapsed time. Repeat it eight times, and calculate the average elapsed time. Then, use the extended locking method to do the same test.

The test results are shown in Table 1 in the single user enviroment. It is easily calculated that the average elapsed time of locking and unlocking one entity is $0.0209 \mathrm{~s}(52.75 / 2525)$ to the old method, and $0.0216 \mathrm{~s}(54.63 / 2525)$ to the new method. The $0.007 \mathrm{~s}(0.0216-0.0209)$ excrescent time of locking and unlocking one spatial entity is few in relative to the elapsed time of its editing produce, which may be lasted for some minutes.

Table 1. The elapsed time using these two method in single user enviroment (Unit:s)

\begin{tabular}{ccc}
\hline Test times & $\begin{array}{l}\text { The elapsed time using } \\
\text { the old method }\end{array}$ & $\begin{array}{l}\text { The elapsed time using } \\
\text { the new method }\end{array}$ \\
\hline 1 & 53 & 54 \\
2 & 52 & 57 \\
3 & 53 & 54 \\
4 & 53 & 55 \\
5 & 54 & 55 \\
6 & 52 & 55 \\
7 & 52 & 53 \\
8 & 53 & 54 \\
Average & 52.75 & 54.63 \\
\hline
\end{tabular}


Test 2: Set up four sessions (multi-user environment), almost simultaneously use the old method to lock and unlock these polygons one by one, and record their elapsed time. Repeat it four times, and calculate the average elapsed time. Then, use the new method to do the same test.

The test results of the old method are shown in Table 2 in the single user enviroment. ' $\infty$ ' stands for the session was enmeshed into deadlocks after one time point. The result show that only session 1 which was start up more ealy would well worked, the others session would enmeshed into deadlocks.

Table 2. The elapsed time using the old method in multi-user enviroment (Unit:s)

\begin{tabular}{ccccc}
\hline Test times & Session 1 & Session 2 & Session 3 & Session 3 \\
\hline 1 & 109 & $\infty$ & $\infty$ & $\infty$ \\
2 & 108 & $\infty$ & $\infty$ & $\infty$ \\
3 & 112 & $\infty$ & $\infty$ & $\infty$ \\
4 & 110 & $\infty$ & $\infty$ & $\infty$ \\
\hline
\end{tabular}

The test results of the new method are shown in Table 3 in single user enviroment. The result show that the four sessions would be worked orderly and finish as soon as possible. The average time elapsed of Table 3 will be quadruple of Table 1, because the four sessions were executed in serial.

Table 3. The elapsed time using the new method in multi-user environment (Unit:s)

\begin{tabular}{ccccc}
\hline Test times & Session 1 & Session 2 & Session 3 & Session 3 \\
\hline 1 & 211 & 215 & 214 & 212 \\
2 & 210 & 212 & 221 & 219 \\
3 & 213 & 216 & 214 & 213 \\
4 & 211 & 215 & 214 & 212 \\
\hline
\end{tabular}

The results of test 2 indicate that the new method is better than the old method as a whole in multi-user environment. The new method keeps the systems work orderly, and the old method makes the most session be in bad deadlock situations.

\section{Conclusions}

This paper has explored an extended locking method for geographical database with spatial rules. With reference to related theories of process management in operation system, the extended locking method implements the management of collaboration and competition between locking and unlocking transactions, avoids deadlocks, and improves the effectiveness of the geographical database management systems. The two tests show that the extended locking technique makes the systems more ordered and efficient in a multi-users environment at the cost of some time consumption of managing logical codes. 
The NCCS is the protocol abided by the extended locking method. Compared with the traditional pessimistic scheme, the NCCS makes the requirements for locking executive less strict and improves the concurrency of the system. While compared with version management, it is easier to be realized and little system expenditure is needed for creating version, recording change, merging two versions, and proposing conflicts.

During the research on geographical database, we should keep in mind the speciality of spatial data and refer theories of computer science to draw conclusions for the use of spatial database.

\section{References}

1. Anthony Chiu, Ben Kao and Kam-yiu Lam: An adaptable constrained locking protocol for high data contention environments: correctness and performance, Information and Software Technology. Vol. 42 (2000) 599-608

2. Bharat Bhargava: Concurrency Control in Database Systems, IEEE Transactions on Knowledge and Data Engineering, Vol. 11 (1999) 3-16.

3. Dean Kuo, Volker Gaede, Kerry Taylor: Using Constrains to Manage Long Duration Transaction in Spatial Information System. http://www.wiwi.hu-berlin.de/ gaede/ltm.ps.gz (19997).

4. Dongman Lee, Jeonghwa Yang, Hee Yong Youn, et al: Entity-Centric Scalable Concurrency Control for Distributed Interactive Applications. Proceedings 19th IEEE Int'1 Performance, Computing, and Communications Conference, (2000)544 550.

5. Zeiler Michael: Modeling Our World: The ESRI Guide to Geodatabase Design. New York: ESRI Press, (2001).

6. E. Easterfield, Richard G. Newell, David G. Theriault: Version Management in GIS Applications and Techniques. Proceedings EGIS ‘90, (1990) 288-297.

7. Gjermund Hanssen: Concurrency control in distributed geographical database systems. Proceedings 9th Scandinavian Research Conference on Geographical Information Science, (2003)167-180.

8. Gopalan Arun: Oracle Workspace Manager: A Framework for Long Transactions. Proceedings GeoSpatial World 2001 Conference,(2001)

9. Jackie Gaskill, Dale Brooks: Understanding ArcSDE: The Gateway to Your RDBMS. Proceedings $12^{\text {th }}$ Annual ESRI International User Conference, (2000).

10. Kevin Mayal, G. Brent Hall: Generalizing GIS: Development of Spatial Grammars for Landscape Planning. http://www.isprs.org/commission4/proceedings/pdfpapers/360.pdf (2002) HYPERLINK

11. Mainguenaud Michel: Consistency of Spatial Database Query Results. Computer, Environment and Urban Systems, Pergamon Press, Vol. 18 (1994)333-342.

12. Manhoi Choy, Mei-Po Kwan, Hong Va Leong: Proceedings the 27th Hawaii International Conference on System Sciences, Vol.IV(1994)337-346.

13. Oracle Corp: Data Concurrency and Consistency. http://downloadwest.oracle.com/docs/cd/B10501_01/server.920/a96524/c21 cnsis.htm\#3178.

14. Peter M. Batty: Version Management Revisited. http://www.gita.org/members_only/downloads/batty.pdf(2002).

15. Prabhudev Konana, Sudha Ram : Transaction management mechanisms for active and real-time databases: A comprehensive protocol and a performance study. The Journal of Systems and Software, Vol. 42, (1998) 205-225 
16. Richard G. Newell: The Why and the How of the Long Transaction. http://emea.smallworld.co.uk/support/techpaper/tp9.html.

17. R. Lea, Y. Honda, K. Matsuda: Virtual Society: Collaboration in 3d Spaces on the Internet. The Journal of Collaborative Computing, Vol. 6 (1997)227-250.

18. Sha Zongyao, Bian Fuling: Comprehensive Knowledge Discovery: Theory, Concept and Application.http://www.isprs.org/commission4/proceedings/pdfpapers/191.pdf(2002).HYP ERLINK

19. S. Goel, B. Bhargava and S. K. Madria: An adaptable constrained locking protocol for high data contention environments: correctness and performance, Information and Software Technology, Vol. 42(2000) 599-608.

20. Thomas Connolly, Carolyn Begg: Database Systems: A Practical Approach to Design, Implementation, and Management 3/e, Addison Wesley Higher Education(2001). 